\title{
Servizio STATISTICO
}

Linistero dell 'Strusione, dell Universitic e della Pricenca

Focus "Le dotazioni multimediali per la didattica nelle scuole"

A.S. $2014 / 15$

(Ottobre 2015) 


\section{Servizio STATISTICO (5)

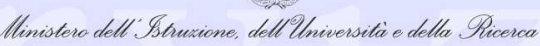

I dati contenuti nella presente pubblicazione fanno riferimento alla data del 14 febbraio 2015.

I dati elaborati e qui pubblicati sono patrimonio della collettività: è consentito il loro utilizzo e la loro pubblicazione con la citazione della fonte ("Fonte: MIUR - ufficio Statistica e studi "; "Fonte: elaborazione su dati MIUR - ufficio Statistica e studi").

La pubblicazione è stata curata da:

Ufficio VI Statistica e studi - D. G. per i contratti, gli acquisti e per i sistemi informativi e la statistica

Ha collaborato:

Ufficio III Supporto per i sistemi informativi, l'innovazione digitale, le analisi statistiche e rapporti internazionali Dipartimento per la programmazione e la gestione delle risorse umane, finanziarie e strumentali 


\section{Servizio STATISTICO

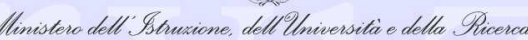

\section{Premessa}

II presente Focus riporta i risultati dell'ultima rilevazione sulle dotazioni tecnologiche presenti nelle scuole, svolta nell'anno scolastico 2014/2015. Obiettivo dell'indagine è quello di rilevare il processo di dematerializzazione di alcuni servizi, le dotazioni tecnologiche, la situazione della connettività di laboratori e aule nonché la diffusione dei dispositivi mobili nelle istituzioni scolastiche.

Il tasso di risposta è stato del $97,6 \%$ per le istituzioni scolastiche statali.

Per la prima volta l'indagine è stata estesa anche alle scuole paritarie. Tuttavia, considerato che il tasso di risposta è stato piuttosto basso $(54,1 \%)$, in questo focus vengono riportati, per questa tipologia di scuole, solo alcuni risultati relativi agli aspetti della dematerializzazione dei servizi.

\section{Maggior utilizzo dei servizi di dematerializzazione}

II processo di digitalizzazione nella Pubblica Amministrazione ha avuto avvio con la legge n.183/2011 (legge di stabilità 2012). Nel settore della scuola tale processo ha avuto una decisa accelerazione con l'entrata in vigore del D.L. n.95/2012 contenente "Disposizioni urgenti per la razionalizzazione della spesa pubblica", convertito dalla legge n.135/2012. Le innovazioni introdotte nella scuola riguardano in particolare i seguenti ambiti:

- le iscrizioni degli studenti, da effettuare con modalità online;

- la pagella in formato elettronico, che ha la medesima validità legale del documento cartaceo ed è

resa disponibile per le famiglie sul web o tramite posta elettronica o altra modalità digitale;

- $\quad$ i registri elettronici del docente e di classe;

- l'invio delle comunicazioni agli alunni e alle famiglie in formato elettronico.

I cambiamenti che si sono prodotti nelle istituzioni scolastiche, in materia di gestione informatizzata dei servizi, possono essere apprezzati attraverso i dati sinteticamente riportati nella tabella 1.

II sito web o portale è ormai disponibile per la quasi totalità degli istituti statali $(99,3 \%)$, mentre lo è di meno nelle scuole paritarie $(67,6 \%)$.

II servizio di comunicazione scuola - famiglia online è attivo nel $58,3 \%$ degli istituti statali e nel $49,7 \%$ di quelli paritari.

II registro elettronico di classe risulta utilizzato nel 69,2\% degli istituti statali e ancor più il registro del docente, presente nel $73,6 \%$ degli istituti. Nelle scuole paritarie questi servizi risultano ancora poco utilizzati (rispettivamente $25,4 \%$ e $24,7 \%$ degli istituti paritari).

Rimane ancora poco diffuso l'utilizzo di piattaforme per la condivisione di materiale didattico e per la gestione di corsi online (LMS -Learning Management System ${ }^{(1)}$ ). Infatti, solo nel 16,5\% degli istituti statali è presente una piattaforma LMS (Graf.1). Da notare che il dato non comprende informazioni sull'utilizzo delle diverse piattaforme presenti online da parte dei singoli docenti. 


\section{Servizio STATISTICO

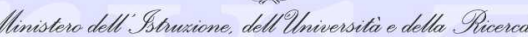

\section{In crescita le tecnologie nella scuola}

Dal lato delle dotazioni tecnologiche per l'insegnamento l'analisi riguarda, come s'è detto, unicamente le scuole statali.

Negli istituti italiani complessivamente sono presenti 65.650 laboratori. Di questi il $43,6 \%$ è dotato di LIM e il $16,9 \%$ di proiettori interattivi, tipologia di dispositivi, quest'ultima, che si sta velocemente affermando come valida alternativa alle LIM, avendo caratteristiche meno vincolate al tipo di hardware e software da utilizzare per la connessione e la proiezione dei contenuti didattici multimediali.

Mediamente ciascun laboratorio dispone di quasi 9 computer. Infine, l' $82,5 \%$ dei laboratori delle scuole statali è collegato in rete (rete cablata o wireless). Occorre tenere presente che i dati sui laboratori si riferiscono a tutte le tipologie di laboratorio e non solo ai professionalizzanti (Tab 2).

Per quel che riguarda le dotazioni presenti nelle aule, il 41,9\% dispone di LIM, il 6,1\% di proiettori interattivi, il $70 \%$ delle aule circa sono connesse in rete (cablata o wireless)(Tab 3). Nell'interpretare quest'ultimo dato va tenuto presente che in numerosi istituti i dispositivi in grado di consentire la connessione alla rete in modalità wireless sono presenti in ambienti comuni degli istituti (es. corridoi), garantendo in tal modo la copertura di diverse aule con un singolo access point.

L'indagine rileva (Tab.4) un deciso incremento delle aule che dispongono della LIM a prescindere dal livello scolastico, a dimostrazione dei forti investimenti negli anni passati. Tali dispositivi, con l'interazione di "dispositivi leggeri" (tablet, smartphone...), darà la possibilità di creare ambienti sempre più flessibili per una didattica digitale.

II quadro fin qui presentato fa riferimento all' anno scolastico 2014/2015. II raffronto con il precedente anno scolastico, 2013-14 (Graf. 3,4,6 e 7), evidenzia un generalizzato incremento delle dotazioni tecnologiche disponibili per la didattica tanto nei laboratori che nelle aule.

Ciò dipende, in primo luogo, dall'impulso che il MIUR, unitamente ad altri soggetti istituzionali, ha assicurato negli ultimi anni per la diffusione delle tecnologie nelle scuole.

A questo specifico riguardo occorre tenere presente che molti dei progetti avviati dal MIUR negli anni precedenti sono giunti a conclusione in questi ultimi due anni: in particolare l'Accordo MIUR Regioni del 2012 per i progetti cofinanziati "LIM in classe", "Classi 2.0", Scuole 2.0" e "Centri scolastici digitali" ed il progetto "Wireless nelle scuole" del 2013.

Inoltre, si sono aggiunti interventi specifici sia da parte di enti locali e territoriali sia da parte di aziende, fondazioni, associazioni culturali $e$ di privati cittadini che hanno contribuito all'incremento/ammodernamento delle dotazioni informatiche.

Infine, nelle regioni dell'Obiettivo Convergenza ${ }^{(2)}$ le scuole hanno beneficiato di contributi per il miglioramento delle dotazioni tecnologiche, finanziati dai fondi strutturali europei (FESR e FSE) nel quadro dei programmi PON-Istruzione e POR nel periodo 2007-2013. Come emerge dal raffronto dei dati (Graf. 6 e 7) riferiti alle ultime due annualità, in Calabria, Campania e Sicilia si riscontra un aumento significativo del numero di aule dotate di LIM e in Puglia delle aule dotate di proiettore interattivo.

\section{Diminuisce il rapporto alunni su tecnologie: più dispositivi in uso agli alunni}

Uno degli indicatori più utilizzati, anche in campo internazionale, per misurare la diffusione delle TIC nelle scuole è il rapporto tra alunni e tecnologie. L'indicatore è calcolato rapportando gli alunni alla somma dei computer presenti nei laboratori e i computer e dispositivi mobili ad uso degli alunni presenti 


\section{Servizio STATISTICO

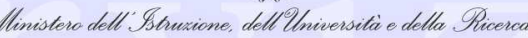

nelle aule. I risultati della rilevazione indicano che la situazione, a livello nazionale, è migliorata passando da 8,9 alunni che utilizzano un singolo device nell'anno scolastico 2013-2014 a 7,9 alunni per device nell'anno scolastico 2014-2015. A livello territoriale si riscontra un miglioramento dell'indicatore soprattutto in Sicilia $(-2,0)$, Lombardia $(-1,6)$, Campania(-1,5) e Calabria $(-1,3)^{(3)}($ Tab.5). Analizzando il rapporto tra gli alunni e ogni singola tecnologia emerge un significativo incremento dei dispositivi mobili in uso agli studenti (da 177 dispositivi mobili per alunno nel 2013/14 a circa 63 del 2014/15) (Graf.8).

L'aumento delle tecnologie leggere non ha riguardato solo i dispositivi in uso agli alunni, come evidenziato nella tabella $5 \mathrm{ma}$ anche quelle a disposizione del docente. Si osserva infatti che il rapporto tra alunno e dispositivo mobile in totale (sia quelli in uso agli alunni che in uso ai docenti)(Tab.6) è passato da 82,2 del 2013/2014 al 32,1 del 2014/2015. Questo forte miglioramento può trovare una giustificazione in una probabile diffusione di una metodologia didattica sempre più collaborativa e partecipativa, nell'ottica di una "classe digitale leggera".

Sebbene le TIC nelle scuole si stiano diffondendo velocemente, l'analisi più particolareggiata a livello locale, evidenzia una situazione caratterizzata da ampi divari, in cui accanto a scuole che dispongono in misura sufficiente o più che sufficiente di tali tecnologie, vi sono scuole contrassegnate da evidente carenza.

Si osserva, infatti, che a livello nazionale a fronte di un $41,1 \%$ di scuole che ha più di 10 computer o dispositivi mobili per la didattica (a uso docenti e/o studenti), il 7,9\% delle scuole dispone di un solo computer per la didattica e il 9,8\% non ne dispone affatto (Graf.9). Sebbene la maggioranza degli studenti, ovvero il $62 \%$, frequenti il $41 \%$ di scuole con più tecnologie in aula, non appare trascurabile la quota del $9,2 \%$ di studenti iscritti presso scuole in cui la didattica in aula non può fruire delle potenzialità delle tecnologie (Tab. 7).

A livello regionale le differenze risultano ancor più marcate. In Basilicata un terzo delle scuole dispone al più di un solo dispositivo, una situazione che coinvolge il $22 \%$ degli studenti della regione. La situazione più favorevole si rileva in Emilia Romagna dove gli alunni che si trovano in scuole poco attrezzate (pari al $7 \%$ ) sono solo il $3 \%$ del totale regionale, mentre il $74 \%$ frequenta le scuole con maggiore dotazione tecnologica.

Note

(1) Gli LMS (learning management system) sono piattaforme in grado di gestire attività di formazione a distanza e condivisione di contenuti. Permettono di seguire tutte le fasi dell'azione formativa: analisi, progettazione, realizzazione, valutazione e monitoraggio. II docente può gestire una classe virtuale condividendo materiale, pianificando attività, somministrando verifiche. Tipici moduli presenti: Area riservata, gestione contenuti didattici, forum, chat, sistema di messaggistica, calendario, monitoraggio delle attività

(2) Le Regioni dell' Obiettivo Convergenza, nel periodo 2007 - 2013, sono la Campania, la Calabria, la Sicilia e la Puglia. Tali regioni rientrano nell'Obiettivo Convergenza dell'Unione Europea perché hanno un PIL pro capite inferiore al $75 \%$ della media comunitaria. L'obiettivo Convergenza è finalizzato ad accelerare lo sviluppo socio economico delle Regioni interessate, migliorando le condizioni per la crescita e l'occupazione attraverso l'aumento e il miglioramento della qualità degli investimenti in capitale fisico e umano, lo sviluppo dell'innovazione e della società della conoscenza, dell'adattabilità ai cambiamenti economici e sociali, la tutela e il miglioramento della qualità dell'ambiente e l'efficienza amministrativa.

(3) I dati relativi all'anno scolastico 2013/2014 rappresentano un aggiornamento di quelli contenuti nel precedente Focus, a seguito del successivo completamento della rilevazione. 


\section{Servizio STATISTICO}

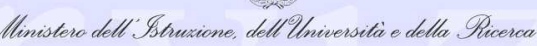

Tab. 1 - La dematerializzazione nelle Istituzioni Scolastiche - A.S. 2014/2015 (valori percentuali)

\begin{tabular}{|c|c|c|c|c|c|c|c|c|}
\hline \multirow{3}{*}{ Regioni } & \multicolumn{8}{|c|}{ Istituzioni scolastiche che hanno dichiarato di avere tra i servizi offerti } \\
\hline & \multicolumn{2}{|c|}{ Sito o portale web } & \multicolumn{2}{|c|}{$\begin{array}{l}\text { Comunicazione scuola-famiglia } \\
\text { online }\end{array}$} & \multicolumn{2}{|c|}{ Registro elettronico di classe } & \multicolumn{2}{|c|}{$\begin{array}{l}\text { Registro elettronico del } \\
\text { docente }\end{array}$} \\
\hline & Statali & Paritarie $(*)$ & Statali & Paritarie $(*)$ & Statali & Paritarie $(*)$ & Statali & Paritarie $(*)$ \\
\hline Piemonte & 98,6 & 71,4 & 58,8 & 55,6 & 72,2 & 37,3 & 74,3 & 39,7 \\
\hline Lombardia & 99,1 & 71,4 & 63,1 & 61,9 & 70,8 & 36,4 & 76,8 & 34,7 \\
\hline Veneto & 99,5 & 59,1 & 56,3 & 48,8 & 72,1 & 37,9 & 80,0 & 36,0 \\
\hline Friuli V.G. & 99,4 & 59,3 & 54,1 & 33,3 & 64,5 & 14,8 & 68,6 & 22,2 \\
\hline Liguria & 99,5 & 65,1 & 67,4 & 46,5 & 73,3 & 18,6 & 72,7 & 14,0 \\
\hline Emilia Romagna & 99,6 & 80,9 & 69,0 & 63,0 & 88,2 & 35,2 & 89,1 & 38,3 \\
\hline Toscana & 99,0 & 71,3 & 67,1 & 51,9 & 68,5 & 16,7 & 76,0 & 17,6 \\
\hline Umbria & 100,0 & 42,9 & 70,9 & 28,6 & 80,9 & 14,3 & 83,7 & 14,3 \\
\hline Marche & 100,0 & 53,6 & 79,8 & 35,7 & 86,8 & 14,3 & 91,8 & 17,9 \\
\hline Lazio & 99,3 & 72,5 & 57,9 & 47,7 & 56,5 & 26,3 & 67,2 & 25,6 \\
\hline Abruzzo & 100,0 & 43,2 & 49,2 & 24,3 & 65,5 & 10,8 & 69,0 & 10,8 \\
\hline Molise & 100,0 & - & 64,3 & - & 76,8 & - & 83,9 & - \\
\hline Campania & 99,3 & 62,4 & 52,8 & 40,2 & 62,6 & 12,7 & 67,3 & 10,1 \\
\hline Puglia & 98,7 & 59,7 & 50,4 & 32,8 & 67,3 & 9,0 & 71,1 & 9,0 \\
\hline Basilicata & 97,9 & 66,7 & 52,9 & 50,0 & 77,9 & 16,7 & 83,6 & 16,7 \\
\hline Calabria & 99,5 & 78,4 & 45,8 & 45,9 & 54,5 & 8,1 & 54,2 & 8,1 \\
\hline Sicilia & 100,0 & 62,2 & 54,4 & 37,8 & 74,6 & 8,0 & 75,8 & 6,9 \\
\hline Sardegna & 98,7 & 63,9 & 53,4 & 41,7 & 55,4 & 16,7 & 55,7 & 16,7 \\
\hline Italia & 99,3 & 67,6 & 58,3 & 49,7 & 69,2 & 25,4 & 73,6 & 24,7 \\
\hline
\end{tabular}

(*) I dati delle scuole paritarie si riferiscono al $54,1 \%$ del totale delle scuole.

Graf.1 - Gestione centralizzata contenuti multimediali LMS - A.S. 2014/2015

(percentuale di Istitutuzioni scolastiche statali che utilizzano la piattaforma LMS)

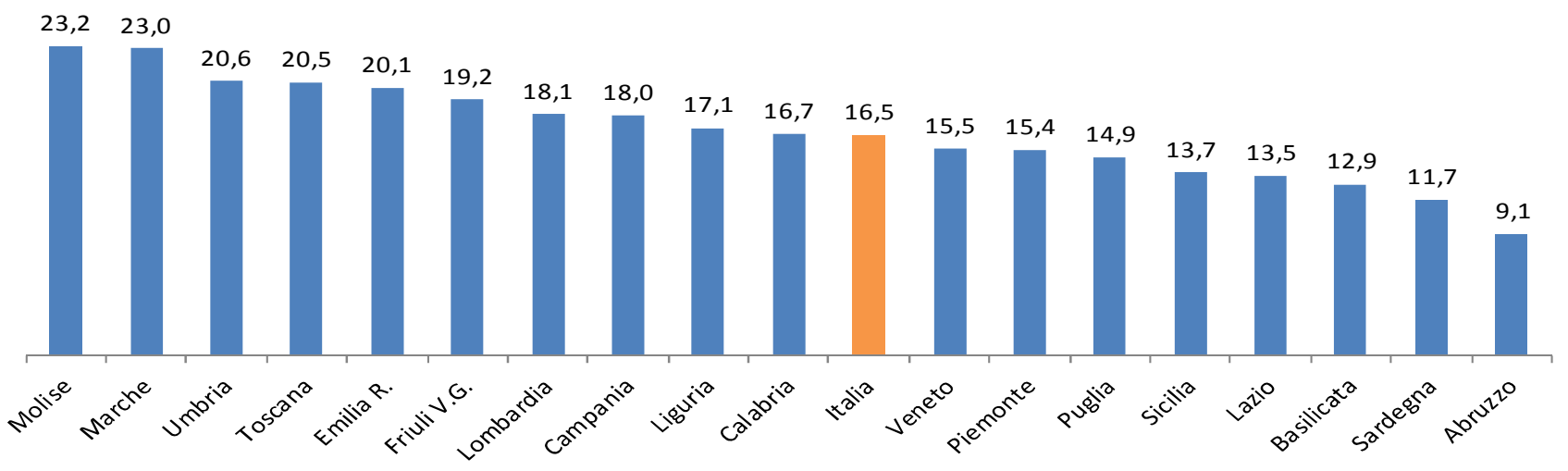


Tab. 2 - Dotazioni tecnologiche nei laboratori delle Istituzioni scolastiche per regione_Scuola statale - A.S. 2014/2015 (valori percentuali)

\begin{tabular}{|c|c|c|c|c|}
\hline \multirow[b]{2}{*}{ Regioni } & \multicolumn{3}{|c|}{ Laboratori } & \multirow{2}{*}{$\begin{array}{r}\text { N. medio di } \\
\text { computer per } \\
\text { laboratorio }\end{array}$} \\
\hline & $\begin{array}{r}\text { connessi in } \\
\text { rete cablata o } \\
\text { wireless }\end{array}$ & $\begin{array}{r}\text { dotati di } \\
\text { LIM }\end{array}$ & $\begin{array}{r}\text { dotati di } \\
\text { proiettore } \\
\text { interattivo }\end{array}$ & \\
\hline Piemonte & 83,0 & 34,3 & 16,1 & 7,2 \\
\hline Lombardia & 82,4 & 36,6 & 16,2 & 8,3 \\
\hline Veneto & 84,5 & 28,7 & 19,3 & 9,5 \\
\hline Friuli V.G. & 84,0 & 31,9 & 15,7 & 8,1 \\
\hline Liguria & 81,3 & 32,1 & 12,8 & 7,2 \\
\hline Emilia Romagna & 88,6 & 30,8 & 19,2 & 7,9 \\
\hline Toscana & 81,3 & 38,2 & 17,0 & 7,4 \\
\hline Umbria & 85,9 & 34,2 & 15,3 & 6,8 \\
\hline Marche & 86,8 & 33,7 & 16,1 & 7,2 \\
\hline Lazio & 79,2 & 49,0 & 18,1 & 8,7 \\
\hline Abruzzo & 82,5 & 32,4 & 17,2 & 7,4 \\
\hline Molise & 83,6 & 35,9 & 18,7 & 8,4 \\
\hline Campania & 80,5 & 61,5 & 16,7 & 9,7 \\
\hline Puglia & 82,8 & 58,3 & 16,9 & 10,4 \\
\hline Basilicata & 85,5 & 44,7 & 16,2 & 7,3 \\
\hline Calabria & 74,5 & 61,4 & 17,6 & 10,1 \\
\hline Sicilia & 83,2 & 56,2 & 16,2 & 9,6 \\
\hline Sardegna & 80,7 & 40,6 & 14,2 & 7,2 \\
\hline Italia & 82,5 & 43,6 & 16,9 & 8,6 \\
\hline Italia 2013-14 & 77,5 & 41,0 & 13,8 & 8,5 \\
\hline
\end{tabular}


Tab. 3 - Dotazioni tecnologiche nelle aule per regione_Scuola statale - A.S. 2014/2015 (valori percentuali)

\begin{tabular}{|c|c|c|c|}
\hline \multirow[b]{2}{*}{ Regioni } & \multicolumn{3}{|c|}{ Aule } \\
\hline & $\begin{array}{r}\text { connesse in } \\
\text { rete cablata o } \\
\text { wireless }\end{array}$ & dotate di LIM & $\begin{array}{r}\text { dotate di } \\
\text { proiettore } \\
\text { interattivo }\end{array}$ \\
\hline Piemonte & 66,1 & 23,3 & 4,9 \\
\hline Lombardia & 73,0 & 37,9 & 8,7 \\
\hline Veneto & 66,0 & 37,1 & 6,2 \\
\hline Friuli V.G. & 64,9 & 26,8 & 6,4 \\
\hline Liguria & 68,5 & 24,9 & 5,5 \\
\hline Emilia Romagna & 81,9 & 42,3 & 8,0 \\
\hline Toscana & 69,4 & 31,4 & 5,8 \\
\hline Umbria & 74,0 & 34,9 & 7,1 \\
\hline Marche & 76,9 & 33,4 & 6,6 \\
\hline Lazio & 63,3 & 30,0 & 5,9 \\
\hline Abruzzo & 70,9 & 26,4 & 4,2 \\
\hline Molise & 66,6 & 34,2 & 6,2 \\
\hline Campania & 69,8 & 58,5 & 4,3 \\
\hline Puglia & 67,6 & 42,2 & 6,9 \\
\hline Basilicata & 67,4 & 22,3 & 5,7 \\
\hline Calabria & 58,5 & 55,6 & 5,0 \\
\hline Sicilia & 72,2 & 62,3 & 4,6 \\
\hline Sardegna & 81,0 & 85,5 & 3,2 \\
\hline Italia & 70,0 & 41,9 & 6,1 \\
\hline Italia 2013/14 & 52,3 & 29,3 & 4,9 \\
\hline
\end{tabular}




\section{Servizio STATISTICO}

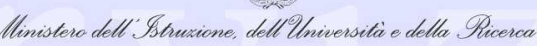

Tab. 4 - Aule dotate di Lim per regione e livello scolastico_Scuola statale - A.S. 2013/2014 - 2014/2015 (valori percentuali)

\begin{tabular}{|c|c|c|c|c|c|c|c|c|}
\hline \multirow{2}{*}{ Regioni } & \multicolumn{2}{|c|}{ Primaria } & \multicolumn{2}{|c|}{ Secondaria I grado } & \multicolumn{2}{|c|}{ Secondaria II grado } & \multicolumn{2}{|c|}{ Totale } \\
\hline & $2013 / 2014$ & $2014 / 2015$ & $2013 / 2014$ & $2014 / 2015$ & $2013 / 2014$ & $2014 / 2015$ & $2013 / 2014$ & $2014 / 2015$ \\
\hline Piemonte & 13,9 & 16,9 & 29,0 & 33,1 & 17,1 & 24,8 & 18,6 & 23,3 \\
\hline Lombardia & 24,9 & 31,6 & 43,9 & 50,2 & 23,1 & 36,9 & 29,2 & 37,9 \\
\hline Veneto & 21,3 & 28,6 & 41,4 & 51,4 & 27,3 & 38,4 & 28,2 & 37,1 \\
\hline Friuli V.G. & 19,5 & 24,7 & 36,0 & 40,8 & 16,0 & 19,3 & 22,3 & 26,8 \\
\hline Liguria & 18,4 & 24,4 & 29,1 & 35,4 & 14,7 & 18,2 & 19,8 & 24,9 \\
\hline Emilia Romagna & 33,0 & 42,9 & 47,3 & 58,7 & 23,7 & 30,3 & 33,2 & 42,3 \\
\hline Toscana & 21,2 & 27,0 & 40,7 & 49,2 & 18,7 & 24,6 & 24,9 & 31,4 \\
\hline Umbria & 22,1 & 29,6 & 50,6 & 58,7 & 20,2 & 26,3 & 27,9 & 34,9 \\
\hline Marche & 27,2 & 32,7 & 44,3 & 53,3 & 15,3 & 21,4 & 27,3 & 33,4 \\
\hline Lazio & 29,5 & 30,8 & 30,2 & 33,8 & 20,6 & 26,4 & 26,6 & 30,0 \\
\hline Abruzzo & 18,7 & 25,8 & 31,7 & 34,8 & 19,4 & 21,5 & 22,1 & 26,4 \\
\hline Molise & 18,4 & 25,0 & 45,7 & 56,7 & 23,8 & 31,0 & 26,9 & 34,2 \\
\hline Campania & 24,2 & 54,5 & 42,5 & 73,0 & 18,4 & 52,6 & 26,8 & 58,5 \\
\hline Puglia & 33,5 & 41,9 & 44,1 & 55,3 & 29,6 & 34,0 & 34,7 & 42,2 \\
\hline Basilicata & 17,0 & 18,5 & 35,6 & 38,3 & 15,3 & 16,1 & 20,9 & 22,3 \\
\hline Calabria & 30,4 & 53,4 & 44,0 & 68,6 & 21,4 & 49,5 & 30,4 & 55,6 \\
\hline Sicilia & 34,3 & 63,2 & 50,2 & 74,9 & 25,2 & 51,5 & 35,2 & 62,3 \\
\hline Sardegna & 70,6 & 84,8 & 77,1 & 86,4 & 69,7 & 85,5 & 71,9 & 85,5 \\
\hline Italia & 26,7 & 38,5 & 42,1 & 54,6 & 23,2 & 37,0 & 29,3 & 41,9 \\
\hline
\end{tabular}




\section{Servizio STATISTICO}

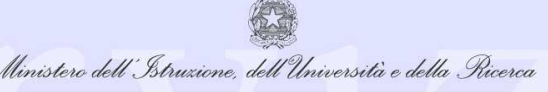

Graf.2 - Laboratori connessi in rete per regione e anno scolastico - Istituti statali (valori percentuali)

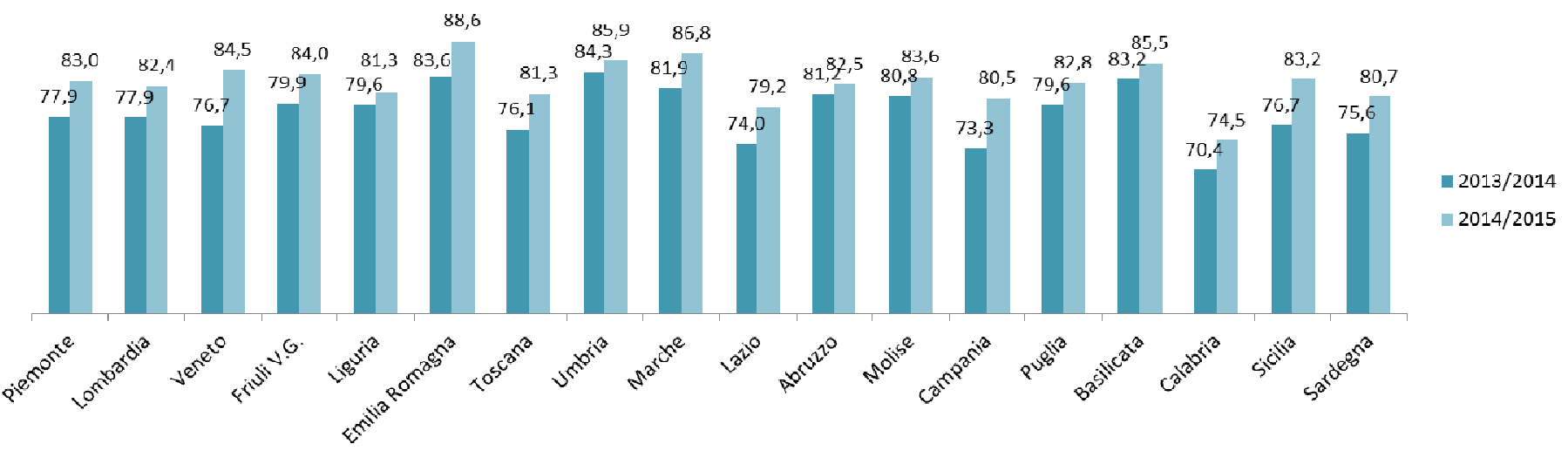

Graf. 3 - Laboratori dotati di LIM per regione e anno scolastico - Istituti statali (valori percentuali)

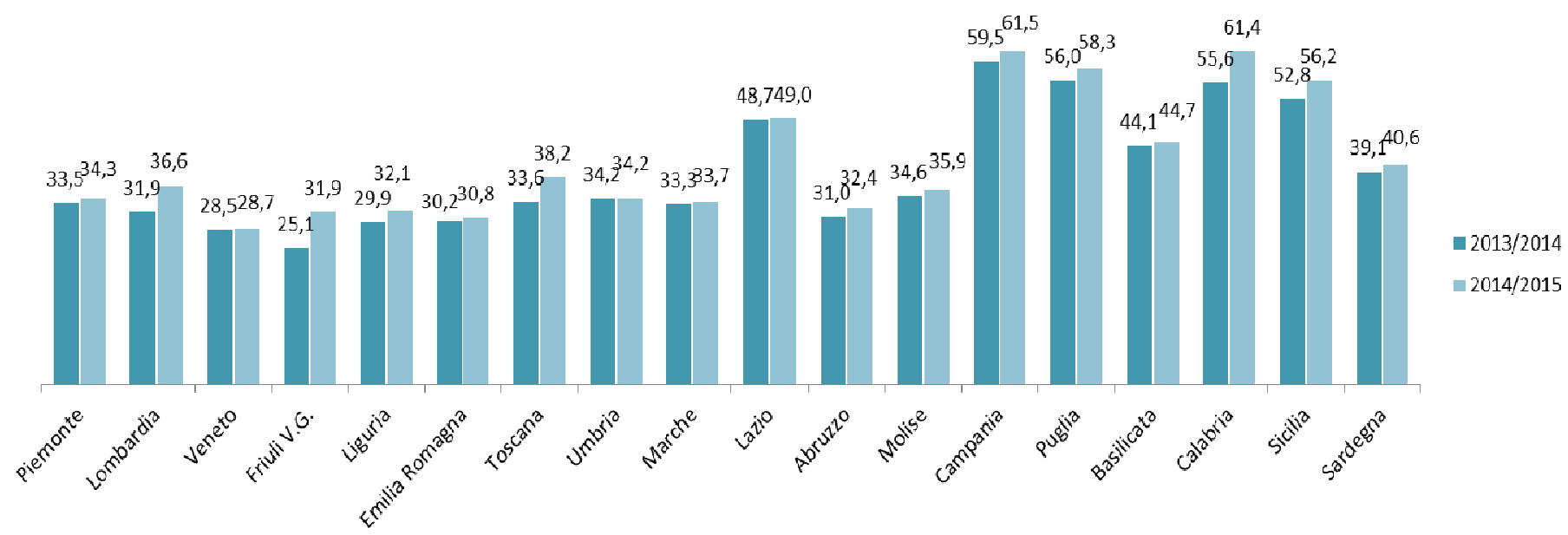

Graf. 4 - Laboratori dotati di proiettori interattivi per regione e anno scolastico - Istituti statali (valori percentuali)

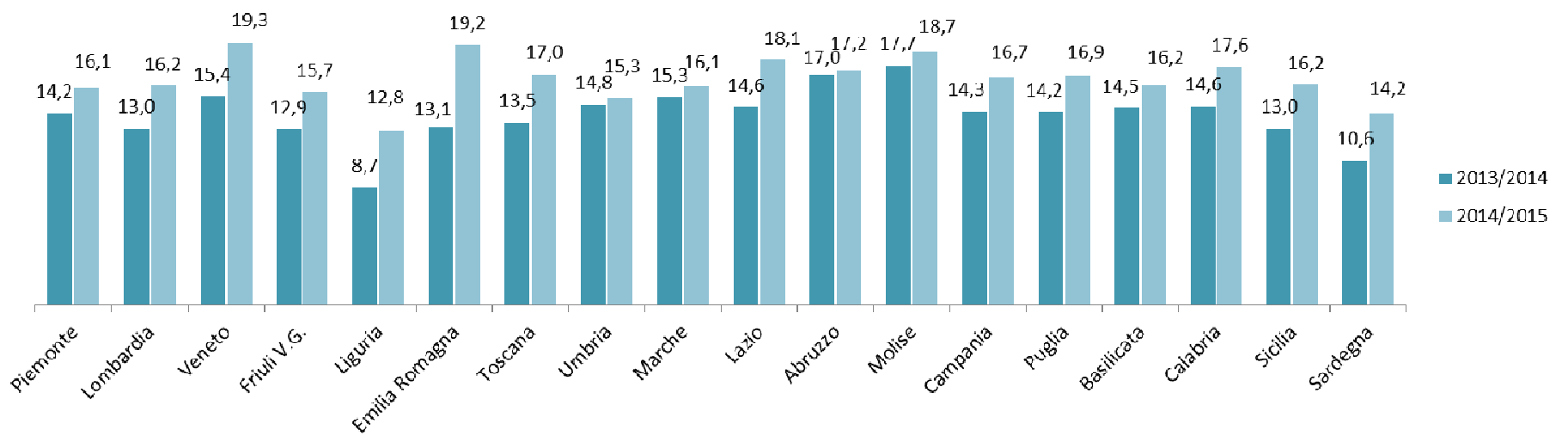




\section{Servizio STATISTICO}

Graf. 5 - Aule connesse in rete per regione e anno scolastico - Scuole statali (valori percentuali)

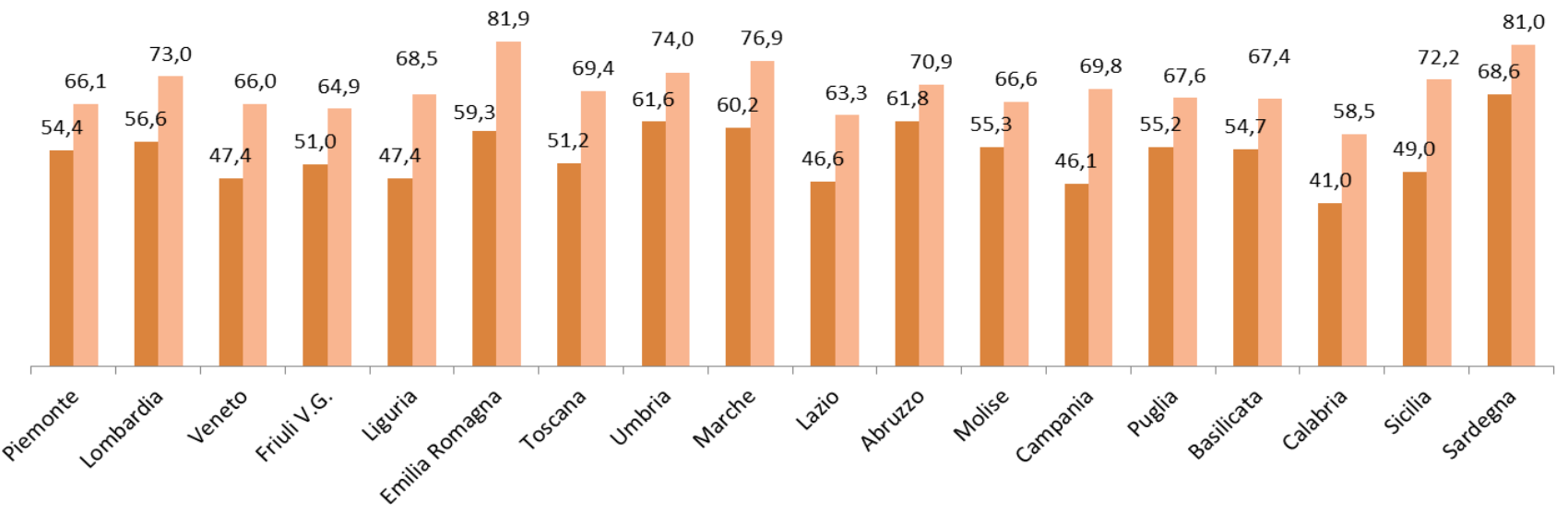

Graf. 6 - Aule dotate di LIM per regione e anno scolastico - Scuole statali (valori percentuali)

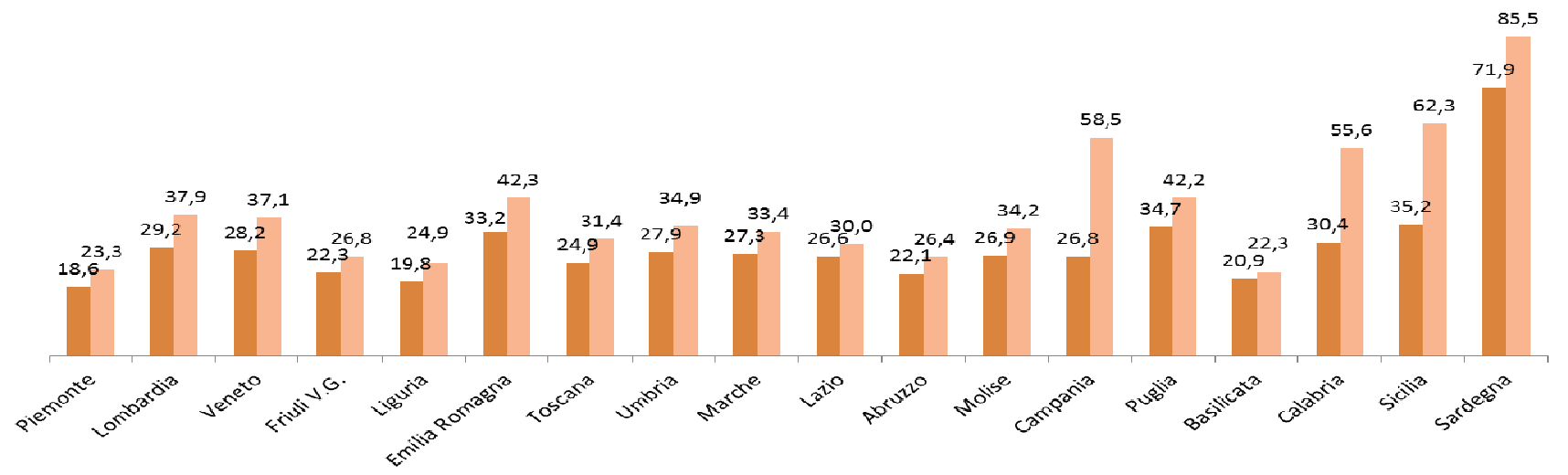

2013/2014

2014/2015

Graf. 7 - Aule dotate di proiettori interattivi per regione e anno scolastico - Scuole statali (valori percentuali)

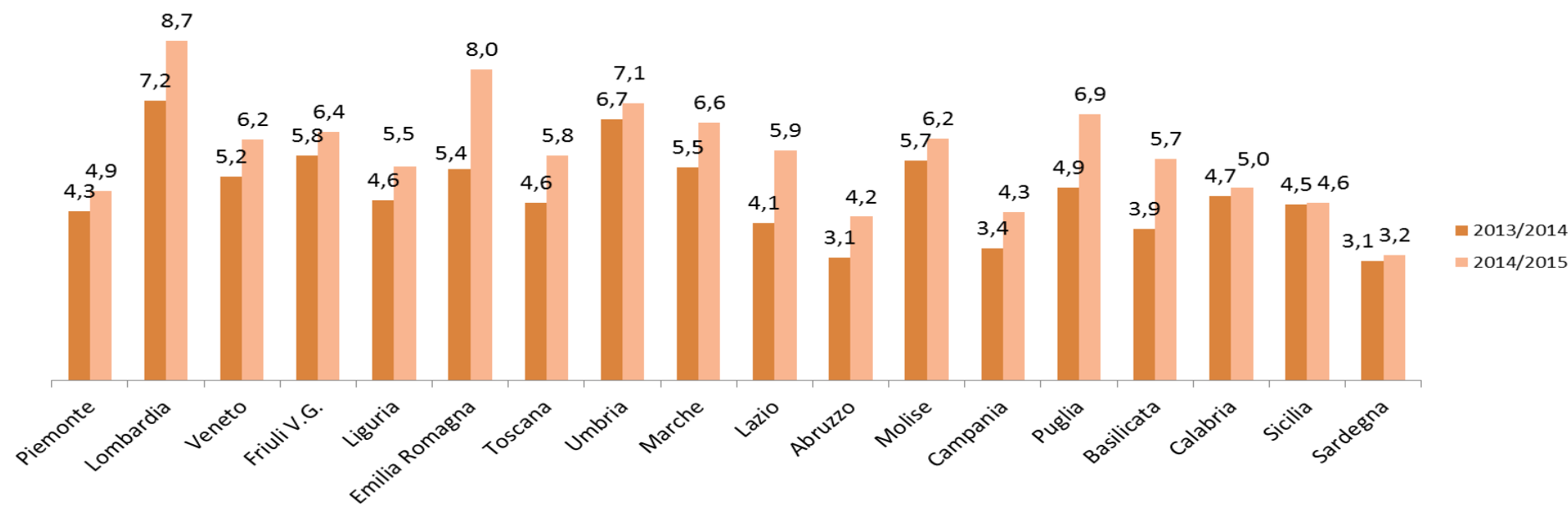




\section{Servizio STATISTICO}

Tab. 5 - Rapporto alunni su tecnologie per regione_Scuola statale - A.S. 2014/2015

\begin{tabular}{|c|c|c|c|c|c|}
\hline \multirow{3}{*}{ Regioni } & \multicolumn{5}{|c|}{ Tecnologie in uso agli alunni } \\
\hline & \multirow{2}{*}{$\begin{array}{r}\text { alunni per } \\
\text { computer in } \\
\text { classe }\end{array}$} & \multirow{2}{*}{$\begin{array}{r}\text { alunni per } \\
\text { dispositivo } \\
\text { mobile }\end{array}$} & \multirow{2}{*}{$\begin{array}{r}\text { alunni per } \\
\text { computer nei } \\
\text { laboratori }\end{array}$} & \multicolumn{2}{|c|}{ alunni su tecnologie } \\
\hline & & & & $2013 / 2014$ & $2014 / 2015$ \\
\hline Piemonte & 39,7 & 215,1 & 12,6 & 9,8 & 9,2 \\
\hline Lombardia & 30,4 & 23,7 & 12,4 & 8,0 & 6,4 \\
\hline Veneto & 50,2 & 228,8 & 10,8 & 8,7 & 8,6 \\
\hline Friuli V.G. & 39,9 & 154,1 & 11,5 & 8,6 & 8,5 \\
\hline Liguria & 29,9 & 77,1 & 12,9 & 8,5 & 8,1 \\
\hline Emilia Romagna & 44,0 & 83,7 & 12,8 & 9,5 & 8,9 \\
\hline Toscana & 49,7 & 96,7 & 14,1 & 10,5 & 9,9 \\
\hline Umbria & 44,6 & 167,0 & 13,0 & 9,9 & 9,5 \\
\hline Marche & 35,0 & 119,3 & 11,3 & 8,5 & 8,0 \\
\hline Lazio & 56,8 & 198,8 & 14,5 & 11,5 & 10,9 \\
\hline Abruzzo & 51,8 & 236,2 & 14,3 & 11,0 & 10,7 \\
\hline Molise & 33,6 & 147,6 & 12,0 & 8,2 & 8,4 \\
\hline Campania & 43,9 & 66,4 & 11,6 & 9,6 & 8,1 \\
\hline Puglia & 43,7 & 160,0 & 9,1 & 7,7 & 7,2 \\
\hline Basilicata & 77,9 & 299,0 & 13,2 & 11,1 & 10,9 \\
\hline Calabria & 36,7 & 37,3 & 7,4 & 6,6 & 5,3 \\
\hline Sicilia & 39,5 & 35,8 & 10,1 & 8,6 & 6,6 \\
\hline Sardegna & 39,9 & 356,9 & 11,0 & 8,6 & 8,4 \\
\hline Italia & 41,0 & 62,8 & 11,6 & 8,9 & 7,9 \\
\hline
\end{tabular}

Graf. 8 - Rapporto alunni su tecnologie_Scuola statale - A.S. 2013/2014 - 2014/2015

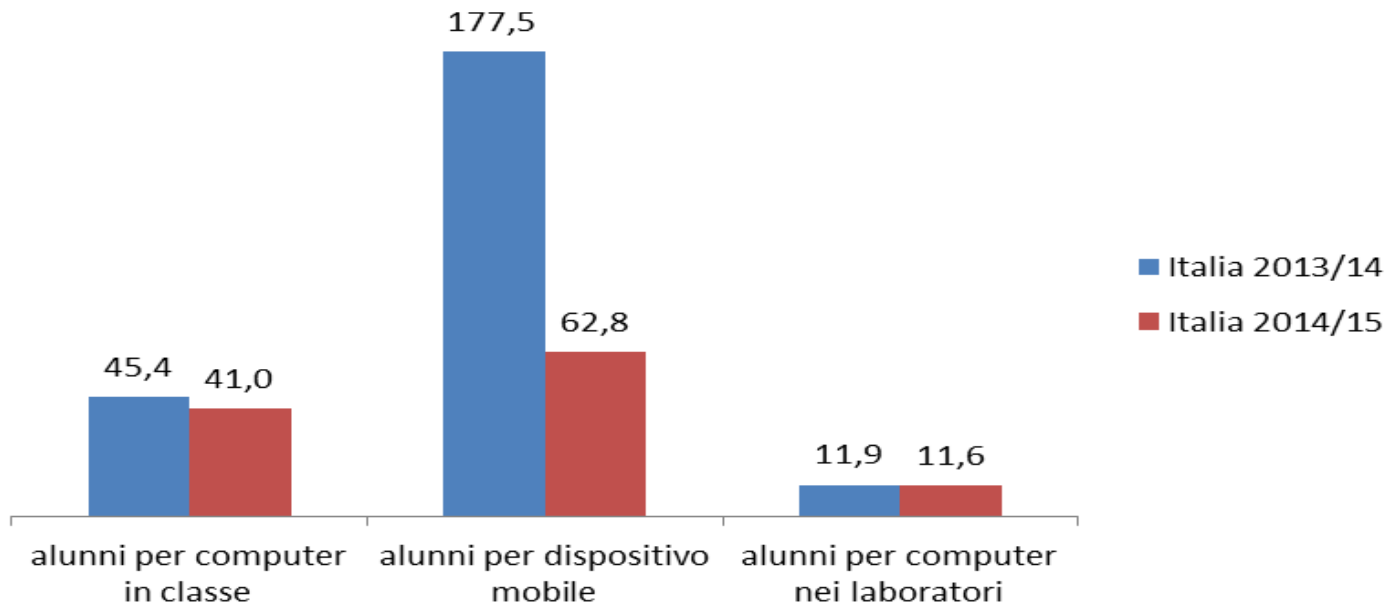




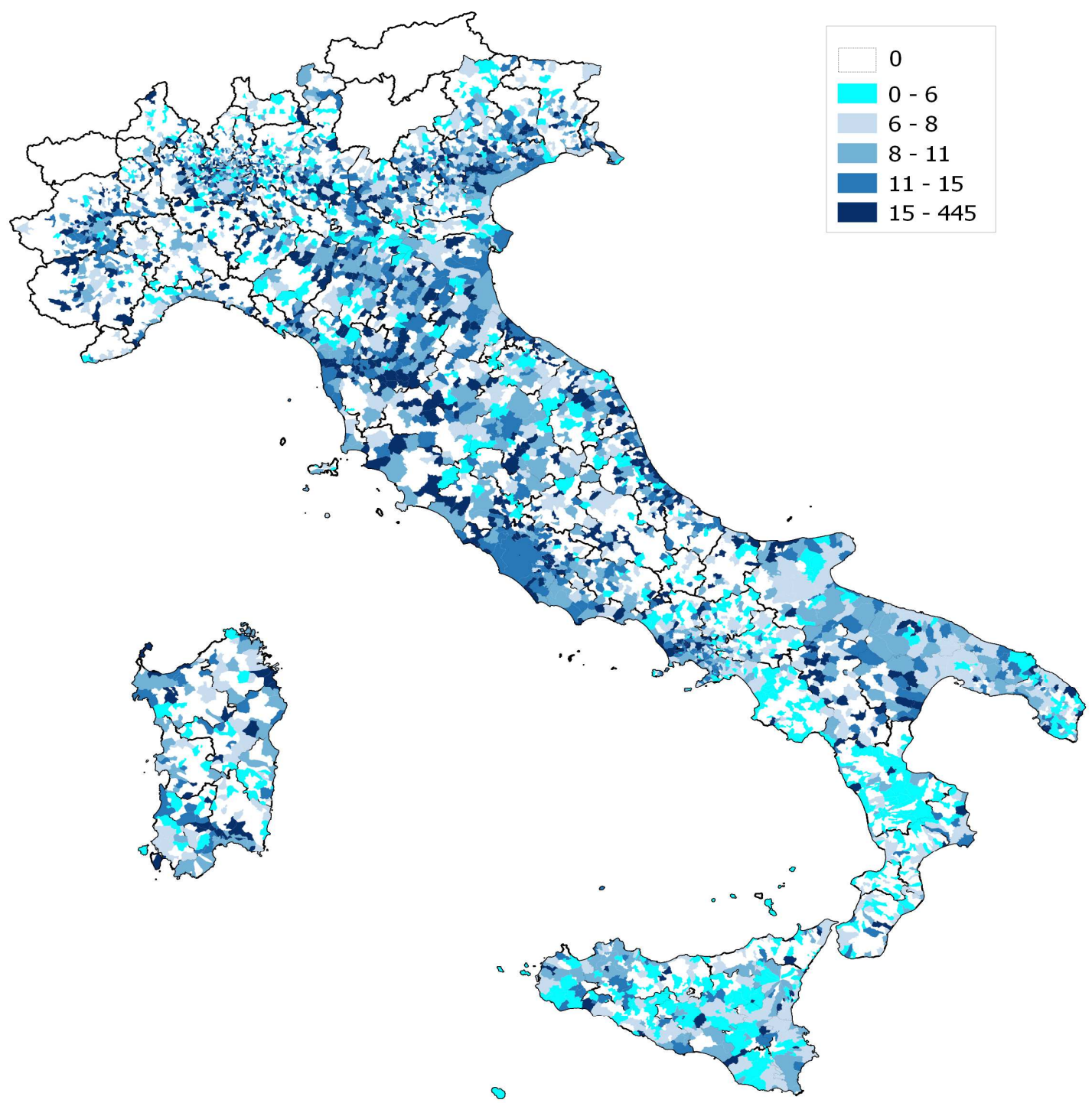

N.B. I dati si riferiscono ai comuni in cui ha sede l'istituto scolastico. Il valore 0 (colore bianco nella legenda) si riferisce a: province autonome di Aosta, Bolzano e Trento le cui scuole non hanno partecipato alla rilevazione; comuni nei quali o non sono presenti istituti scolastici o sono presenti ma non hanno partecipato alla rilevazione. 


\section{Servizio STATISTICO}

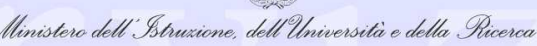

Tab. 6 - Rapporto alunni su totale dispositivi mobili nelle scuole per regione e livello scolastico_Scuola statale - A.S. 2013/2014 2014/2015

\begin{tabular}{|c|c|c|c|c|c|c|c|c|}
\hline \multirow{2}{*}{ Regioni } & \multicolumn{2}{|c|}{ Primaria } & \multicolumn{2}{|c|}{ Secondaria I grado } & \multicolumn{2}{|c|}{ Secondaria II grado } & \multicolumn{2}{|c|}{ Totale } \\
\hline & $2013 / 2014$ & $2014 / 2015$ & $2013 / 2014$ & $2014 / 2015$ & $2013 / 2014$ & $2014 / 2015$ & $2013 / 2014$ & $2014 / 2015$ \\
\hline Piemonte & 228,6 & 114,1 & 124,5 & 59,0 & 71,3 & 43,4 & 116,4 & 63,5 \\
\hline Lombardia & 231,5 & 141,1 & 197,2 & 110,6 & 13,2 & 6,4 & 36,9 & 17,6 \\
\hline Veneto & 414,7 & 147,5 & 167,2 & 66,4 & 56,4 & 33,2 & 117,8 & 59,2 \\
\hline Friuli V.G. & 203,4 & 101,2 & 109,1 & 51,5 & 69,5 & 30,2 & 108,5 & 48,6 \\
\hline Liguria & 195,9 & 98,4 & 55,2 & 36,7 & 41,0 & 35,3 & 65,0 & 47,1 \\
\hline Emilia Romagna & 159,5 & 77,3 & 92,6 & 39,5 & 47,1 & 27,5 & 78,8 & 41,0 \\
\hline Toscana & 303,8 & 88,4 & 74,6 & 34,2 & 55,6 & 30,0 & 89,4 & 42,0 \\
\hline Umbria & 934,5 & 143,2 & 131,6 & 52,9 & 103,0 & 57,4 & 171,9 & 73,1 \\
\hline Marche & 354,4 & 146,6 & 97,1 & 43,8 & 50,2 & 33,6 & 91,1 & 51,3 \\
\hline Lazio & 354,7 & 196,7 & 205,4 & 80,3 & 56,7 & 45,2 & 117,3 & 75,8 \\
\hline Abruzzo & 283,0 & 161,0 & 170,4 & 95,5 & 59,0 & 33,0 & 107,9 & 60,4 \\
\hline Molise & 203,8 & 164,1 & 298,3 & 63,6 & 88,0 & 51,4 & 138,6 & 71,8 \\
\hline Campania & 174,2 & 37,8 & 130,5 & 24,3 & 115,6 & 23,9 & 136,7 & 27,6 \\
\hline Puglia & 176,6 & 62,8 & 103,2 & 38,5 & 72,7 & 31,3 & 103,4 & 40,6 \\
\hline Basilicata & 858,7 & 409,4 & 208,5 & 145,7 & 108,0 & 69,7 & 188,7 & 117,9 \\
\hline Calabria & 174,3 & 31,0 & 81,2 & 14,0 & 46,8 & 15,7 & 74,4 & 18,5 \\
\hline Sicilia & 180,8 & 26,4 & 91,0 & 16,8 & 63,6 & 14,1 & 94,3 & 18,2 \\
\hline Sardegna & $1.184,6$ & 652,1 & 485,0 & 199,3 & 540,6 & 99,9 & 645,6 & 175,0 \\
\hline Italia & 230,0 & 71,5 & 121,3 & 38,6 & 42,4 & 18,9 & 82,2 & 32,1 \\
\hline
\end{tabular}




\section{Servizio STATISTICO}

Graf. 9 - Distribuzione delle scuole per numero di computer e dispositivi mobili_Scuola Statale - A.S. 2014/15 (valori percentuali)

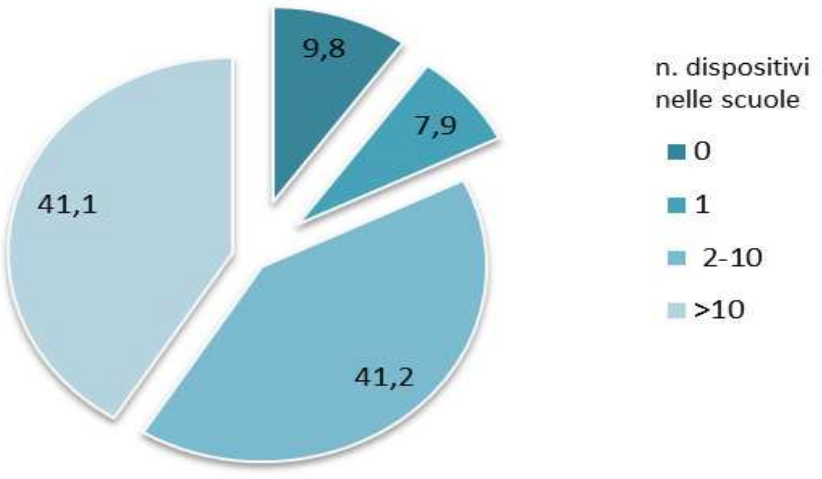

Tab. 7 - Distribuzione delle scuole e degli alunni per numero di computer e dispositivi mobili presenti nelle scuole e per regione_Scuola Statale - A.S. 2014/15 (valori percentuali)

\begin{tabular}{|c|c|c|c|c|}
\hline \multirow[t]{3}{*}{ Regioni } & \multicolumn{4}{|c|}{ computer/dispositivi presenti nella scuola } \\
\hline & \multicolumn{2}{|c|}{$0-1$} & \multicolumn{2}{|c|}{$>10$} \\
\hline & scuole & alunni & scuole & alunni \\
\hline Piemonte & 18,8 & 9,5 & 32,8 & 54,0 \\
\hline Lombardia & 12,5 & 7,3 & 45,1 & 63,7 \\
\hline Veneto & 19,2 & 11,1 & 35,0 & 54,8 \\
\hline Friuli V.G. & 12,3 & 5,8 & 40,1 & 61,5 \\
\hline Liguria & 14,5 & 7,4 & 36,7 & 58,5 \\
\hline Emilia Romagna & 7,1 & 3,3 & 52,1 & 73,5 \\
\hline Toscana & 16,7 & 9,4 & 37,3 & 58,1 \\
\hline Umbria & 18,6 & 8,7 & 33,7 & 60,3 \\
\hline Marche & 11,7 & 5,9 & 37,4 & 57,6 \\
\hline Lazio & 21,5 & 12,3 & 37,2 & 55,6 \\
\hline Abruzzo & 22,2 & 10,6 & 26,6 & 48,4 \\
\hline Molise & 31,9 & 13,8 & 18,1 & 44,7 \\
\hline Campania & 21,3 & 10,4 & 48,2 & 69,0 \\
\hline Puglia & 16,1 & 9,6 & 54,4 & 68,5 \\
\hline Basilicata & 33,3 & 22,2 & 15,6 & 29,2 \\
\hline Calabria & 30,5 & 15,8 & 36,9 & 60,4 \\
\hline Sicilia & 14,5 & 7,6 & 52,8 & 71,7 \\
\hline Sardegna & 19,3 & 9,7 & 28,7 & 52,0 \\
\hline Italia & 17,7 & 9,2 & 41,1 & 62,0 \\
\hline
\end{tabular}

